\title{
Hunting for superheavy dark matter with the highest-energy cosmic rays
}

\author{
Esteban Alcantara, ${ }^{1}$ Luis A. Anchordoqui, ${ }^{1,2,3}$ and Jorge F. Soriano ${ }^{1}$ \\ ${ }^{1}$ Department of Physics and Astronomy, Lehman College, City University of New York, \\ New York 10468, USA \\ ${ }^{2}$ Department of Physics, Graduate Center, City University of New York, New York 10016, USA \\ ${ }^{3}$ Department of Astrophysics, American Museum of Natural History, New York 10024, USA
}

(Received 26 March 2019; published 24 May 2019)

In 15 years of data taking, the Pierre Auger Observatory has observed no events beyond $10^{11.3} \mathrm{GeV}$. This null result translates into an upper bound on the flux of ultrahigh-energy cosmic rays, implying $J\left(>10^{11.3} \mathrm{GeV}\right)<3.6 \times 10^{-5} \mathrm{~km}^{-2} \mathrm{sr}^{-1} \mathrm{yr}^{-1}$, at the $90 \%$ C.L. We interpret this bound as a constraint on extreme-energy photons originating in the decay super-heavy dark matter (SHDM) particles clustered in the Galactic halo. Armed with this constraint, we derive the strongest lower limit on the lifetime of hadronically decaying SHDM particles with masses in the range $10^{14} \lesssim M_{X} / \mathrm{GeV} \lesssim 10^{16}$. We also explore the capability of NASA's future Probe of Extreme Multi-Messenger Astrophysics mission to search for SHDM signals.

DOI: 10.1103/PhysRevD.99.103016

\section{INTRODUCTION}

For the time being, a sovereign objective of the particle physics program is to ascertain the connection between dark matter (DM) and the Standard Model (SM). Existing data constrain the majority of DM to be nonbaryonic, cold or warm, and stable or long lived [1]. There are many ways to accommodate these constraints, and so feasible DM candidates with a very large range of masses and interaction strengths have been proposed [2].

For many decades, the favored models characterized the DM as a relic density of weakly interacting massive particles (WIMPs) [3-6]. ${ }^{1}$ However, LHC experiments have run extensive physics searches for WIMP signals which have returned only null results $[11,12]$. In addition, a broad WIMP search program has been developed with direct and indirect detection methods, which so far have given unsatisfactory answers [13-24]. Despite the fact that a complete exploration of the WIMP parameter space remains the highest priority of the DM community, there is now a strong motivation to explore alternatives to the WIMP paradigm.

Among the well-motivated ideas for what DM could be, the WIMPzilla hypothesis postulates that DM is made of gravitationally produced (nonthermal relic) superweakly

\footnotetext{
${ }^{1}$ For a precise calculation of the WIMP relic abundance, see $[7,8]$; partial wave unitarity dictates an upper bound on the WIMP mass $\leq 110 \mathrm{TeV}[9,10]$.

Published by the American Physical Society under the terms of the Creative Commons Attribution 4.0 International license. Further distribution of this work must maintain attribution to the author(s) and the published article's title, journal citation, and DOI. Funded by SCOAP ${ }^{3}$.
}

interacting supermassive $X$-particles [25-33]. As a matter of fact, the gravitational production of super-heavy dark matter (SHDM) at the end of inflation may be taken as the only experimentally verified DM production mechanism because the observed cosmic microwave background (CMB) fluctuations have precisely the same origin. At the end of inflation, a fraction of fluctuations is not stretched beyond the horizon but remains as $X$-particles because the inflation slows down. The weakness of the gravitational interaction naturally explains the tiny initial abundance of WIMPzillas. Indeed, for such an abundance to be cosmologically relevant today, the $X$-particles must be supermassive.

On an entirely separate though somewhat related note, the surprising absence of any signals of new physics at the LHC experiments [34] seems to indicate that nature does not care too much about our notion of naturalness. Indeed, the required fine-tuning of SM fundamental parameters to accommodate the 15 orders of magnitude between the electroweak and the Planck scales may soon become a reality. Of course, the only reason one may try to incorporate such a shocking idea is that the existence of life may actually be contingent on this wicked conspiracy [35]. Namely, the weak and QCD scales come about just very close to one another, so that a plethora of atoms can exist to exchange energy over extremely long timescales, assembling the building blocks for life and durable habitats where it can thrive [36-39]. ${ }^{2}$ An additional, though not so severe,

\footnotetext{
${ }^{2}$ Investigations in string theory have applied a statistical approach to the enormous "landscape" of vacua present in the theory [37]. Remarkably, this huge number of metastable vacua, $\mathcal{O}\left(10^{500}\right)$, can also accommodate the more severe fine-tuning required to characterize the SM with a small cosmological constant $[40,41]$.
} 
anthropic argument applies to the abundance of DM, which cannot be too much larger or smaller than what is observed [42-45]. This is because DM plays a critical role in structure formation. Note that, since DM is only subject to the force of gravity, the gravitational Jeans instability which allows compact structures to form is not opposed by any force, such as radiation pressure. As a result, DM begins to collapse into a complex network of DM halos well before baryonic matter, which is impeded by pressure forces. Without DM, the epoch of galaxy formation would occur substantially later in the universe than is observed, and consequently the galaxies needed for our existence would not have formed in time. However, it is only the DM abundance and not any other details of the dark sector which is critical for life to exist. Therefore, it is quite reasonable to expect that the DM sector would not be as finely tuned as the visible SM sector. In other words, even if we are prepared to advocate the anthropic argument to accommodate the unnaturalness of the weak scale, we would expect the DM particle spectrum to be as natural as possible, i.e., near the Planck scale that is the natural ultraviolet cutoff scale. For the most part, the WIMPzilla could then be a natural DM candidate and perhaps as well motivated as the WIMP paradigm.

Furthermore, precision CMB measurements enable a direct experimental test of the WIMPzilla hypothesis. This is because the production of SHDM during inflation gives rise to isocurvature perturbations that become sources of gravitational potential energy contributing to the tensor power spectrum of the CMB [46]. This implies a detectable primordial tensor-to-scalar ratio $r$ in the CMB power spectrum. The combined (Planck satellite [47] together with BICEP2 and the Keck array [48]) 95\% C.L. upper bound, $r<0.07$, already constrains the $X$-particle mass to be $M_{X} \lesssim 10^{17} \mathrm{GeV}$ in the limit of instantaneous reheating [49]. For slightly less efficient reheating, this upper limit strengthens to $M_{X} \lesssim 10^{16} \mathrm{GeV}$.

Note also that, while the WIMPzilla must be stable over cosmological timescales, instanton decays induced by operators involving both the hidden sector and the SM sector may give rise to observable signals in the spectrum of ultrahigh-energy cosmic rays (UHECRs) [50,51]. More concretely, the spectrum from WIMPzilla decay is expected to be dominated by photons and neutrinos because of a more effective production of pions than nucleons in the QCD cascades. Since the photons would not be attenuated owing to their proximity, they become the prime signal because it is easier to detect photons than neutrinos. In this article, we use the most recent UHECR data to derive the strongest lower limit on the lifetime of hadronically decaying WIMPzillas. We also investigate the prospects for next generation UHECR experiments to search for SHDM signals.

\section{NEW LIMIT ON THE LIFETIME OF SHDM}

The Pierre Auger Observatory has collected an exposure $\mathcal{E}=67000 \mathrm{~km}^{2}$ sryr without observation of any events with energy $E_{0}>10^{11.3} \mathrm{GeV}$ [52]. This null result sets a generic upper limit on the integrated flux of UHECRs; namely,

$$
\begin{aligned}
J\left(>E_{0}\right)= & \int_{E_{0}}^{\infty} J(E) d E<2.44 / \mathcal{E} \\
& <3.6 \times 10^{-5} \mathrm{~km}^{-2} \mathrm{sr}^{-1} \mathrm{yr}^{-1}
\end{aligned}
$$

at the $90 \%$ C.L.; the limit is a factor of 1.266 less restrictive at the $95 \%$ C.L. [53]. When interpreted as a bound on extreme-energy photons and compared with existing bounds [54,55], this limit is more restrictive by about an order of magnitude, but at a slighter higher energy. Consequently, the all-particle limit of (1) could provide a better weapon to constrain WIMPzilla decay.

To estimate the photon flux from WIMPzilla decay, we need to evaluate two separate contributions: the astrophysical factor and the particle physics factor:

(1) The astrophysical factor is determined by the distribution of DM particles in the Galaxy. The DM density of $X$-particles is a function of the distance $r$ from the Galactic Center and is usually described by a smooth profile function

$$
\rho_{\mathrm{DM}}(r)=\frac{\rho_{s}}{\left[1-\beta+\left(r / r_{s}\right)^{\alpha}\right]\left(1+r / r_{s}\right)^{3-\alpha}},
$$

where $\rho_{s}$ and $r_{s}$ are respectively the scale density and scale radius. The traditional benchmark choice, motivated by N-body simulations, is the NavarroFrenk-White (NFW) profile, in which $\alpha=1, \beta=1$, and $r_{s}=24.42 \mathrm{kpc}$ [56]. The latest numerical simulations, however, seem to favor the Einasto profile,

$$
\rho_{\mathrm{DM}}(r)=\rho_{s} \exp \left\{-\frac{2}{0.17}\left[\left(\frac{r}{r_{s}}\right)^{0.17}-1\right]\right\}
$$

which does not converge to a power law at the Galactic Center and becomes more chubby than NFW at kiloparsec scales, and where $r_{s}=28.44 \mathrm{kpc}$ $[57,58]$. On the other hand, the cored profile put forward by Burkert, for which $\beta=0, \alpha=2$, and $r_{s}=12.67$, is motivated by observations of galactic rotation curves [59]. Profiles steeper than the NFW have also been considered, e.g., the one by Moore and collaborators taking $\alpha=1.16, \beta=1$, and $r_{s}=$ $30.28 \mathrm{kpc}$ [60]. Herein, we take $\rho_{X}=\rho_{\text {DM }}$ and normalize to the local (solar) DM density, $\rho_{X}\left(r_{\odot}\right)=$ $\rho_{\odot}^{\mathrm{DM}}=0.3 \mathrm{GeV} / \mathrm{cm}^{3}$, where $r_{\odot}=8.33 \mathrm{kpc}$ is the distance between the Earth and the Galactic Center [61]. This leads to $\rho_{s} /\left(\mathrm{GeV} \mathrm{cm}^{-3}\right)=0.184,0.033$, 
$\theta$

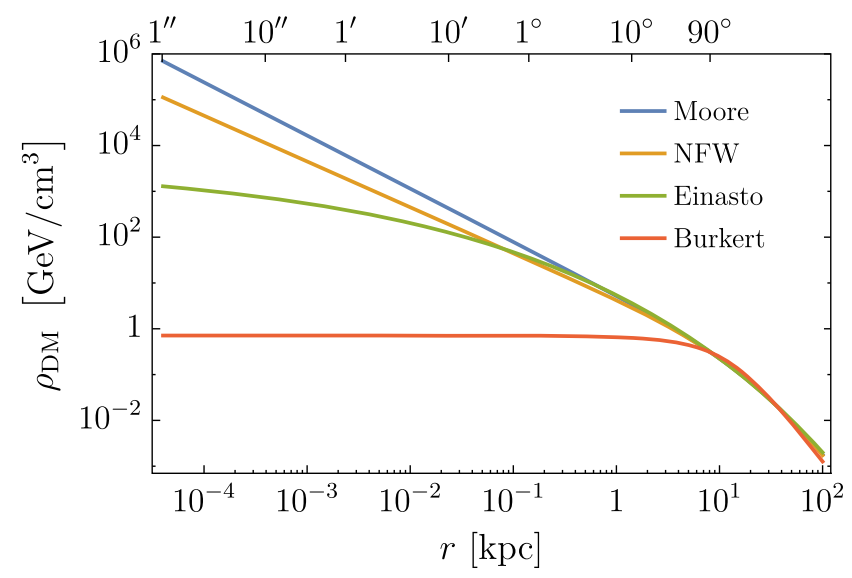

FIG. 1. DM halo mass profiles. The upper horizontal axis shows the variation of the angle between the line of sight and the axis defined by Earth and the Galactic Center.

$0.712,0.105$ for the profiles proposed by NFW, Einasto, Burket, and Moore, respectively [62]. A comparison of these profiles is given in Fig. 1. The ensuing discussion will be framed in the context of NFW, and we will comment on the other profiles after presenting our results.

(2) The particle physics factor is built in the fragmentation function of the SM particles produced by the $X$-decay. There is general agreement among the various computational schemes (relying on either analytic approximations [63] or else Monte Carlo simulations [64-67]) proposed to describe the secondary spectra of SM particles produced via $X$ decay. Herein, we obtain the final state stable particle spectra by solving the Dokshitzer-Gribov-LipatovAltarelli-Parisi equations numerically [68-71]. For an illustration, in Fig. 2, we show the resulting photon, proton, and neutrino $(\nu+\bar{\nu})$ spectra from $X \rightarrow q \bar{q}$ decay. From the observational perspective, the salient

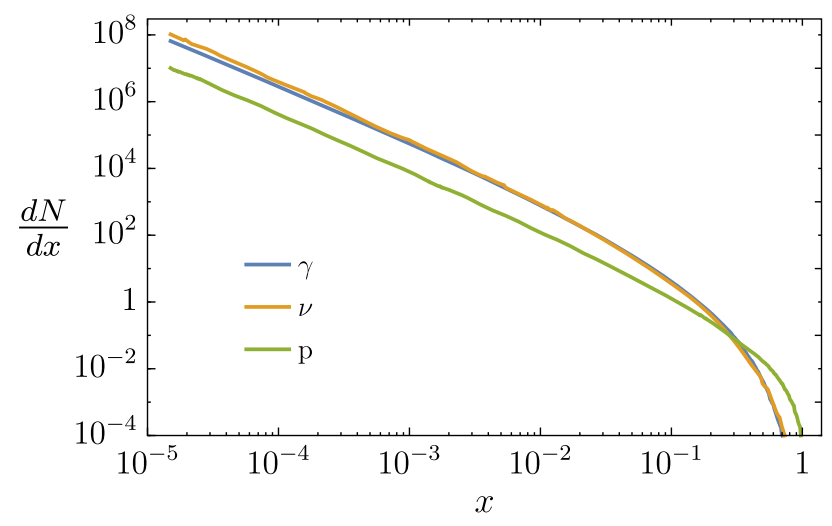

FIG. 2. Spectra of photons, protons, and neutrinos $(\nu+\bar{\nu})$ from $X$-particle decay as a function of the dimensionless variable $x=2 E / M_{X}$. We have taken $M_{X}=10^{16} \mathrm{GeV}$. features of the final state particles (photons, nucleons, and neutrinos) can be summarized as follows:

(a) the spectrum is flat $\left(d N / d E \propto E^{-1.9}\right)$ and independent of the particle type,

(b) the photon/nucleon ratio is $2 \lesssim \gamma / N \lesssim 3$, and the neutrino/nucleon ratio is $3 \lesssim \nu / N \lesssim 4$, both of these ratios being quite independent of the energy.

The expected energy distribution on Earth follows the initial decay spectrum, whereas the angular distribution incorporates the (uncertain) distribution of dark matter in the Galactic halo via the line-of-sight integral [72-75]. The photon flux observed on Earth can be written as

$$
\begin{aligned}
J(E, \theta)= & \frac{1}{4 \pi} \frac{1}{\tau_{X} M_{X}} \frac{d N}{d E}\left\{2 \int_{r_{\odot} \sin \theta}^{r_{\odot}} d r r \frac{\rho_{X}(r)}{\sqrt{r^{2}-r_{\odot}^{2} \sin ^{2} \theta}}\right. \\
& \left.+\int_{r_{\odot}}^{R_{H}} d r r \frac{\rho_{X}(r)}{\sqrt{r^{2}-r_{\odot}^{2} \sin ^{2} \theta}}\right\},
\end{aligned}
$$

where $\theta$ is the angle between the line of sight and the axis defined by Earth and the Galactic Center [76]. Here, $\tau_{X}$ is the WIMPzilla lifetime, and $R_{H}=260 \mathrm{kpc}$ is the radius of the Galactic halo.

Following Ref. [77], we normalize the flux integrating over the whole sky $(0<\theta<\pi)$ and averaging over the directional exposure at the declination of the Pierre Auger Observatory [78]. For $M_{X}=1.7 \times 10^{16} \mathrm{GeV}$ and $\tau_{X}=8.3 \times 10^{21} \mathrm{yr}$, the integral flux of photons at the location of the Pierre Auger Observatory is $J\left(>E_{0}\right)=$ $1.6 \times 10^{-4} \mathrm{~km}^{-2} \mathrm{yr}^{-1} \mathrm{sr}^{-1}$ [79]. This is a factor of 1.75 times smaller than the integral flux of photons derived in Ref. [80] for the same value of $M_{X}$ and $\tau_{X}$, using $\alpha=3 / 2$, $\beta=1$, and $r_{s}=45 \mathrm{Mpc}$ as obtained in Ref. [81]. Now, we compare the integral flux with the upper limit derived in (1) to constrain the $\tau_{X}-M_{X}$ parameter space. Our results are encapsulated in Fig. 3. The growth of the final state stable particle spectra with decreasing $x$ determines the functional form of the constraint on $\tau_{X}$. For masses in the range, $10^{14} \lesssim M_{X} / \mathrm{GeV} \lesssim 10^{16}$, the lower limit (95\% C.L.) on the lifetime of SHDM particles derived in this work is a factor $\gtrsim 2$ more restrictive than previous bounds [77]; see also Refs. [82-84]. For $M_{X} \lesssim 10^{14} \mathrm{GeV}$, constraints on the diffuse photon flux below $E_{0}[54,55]$ provide the most restrictive bound on $\tau_{X}$ [85]. A point worth noting at this juncture is that the limit on $\tau_{X}$ is completely independent of the $X$-production mechanism, and consequently it applies to all SHDM models, e.g., Refs. $[49,86]$.

There are a few caveats to our calculation. On the one hand, it is important to emphasize that the limit derived in Fig. 3 is calculated under the assumption that the photonto-baryon relative exposure of the Auger surface detector array is equal to 1 . This overly simplified assumption may overestimate the actual photon exposure $[87,88]$. We defer 


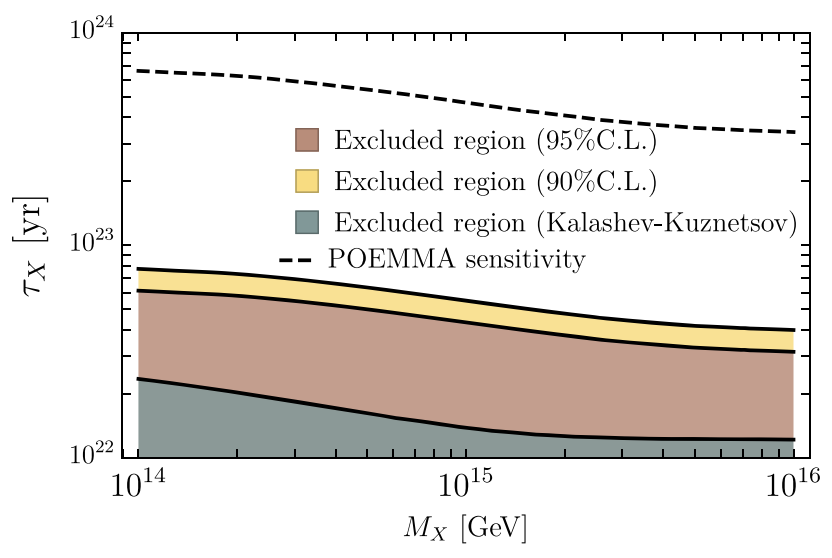

FIG. 3. Lower limit on the lifetime of SHDM particles together with the stereoscopic $\tau_{X}$ sensitivity (defined by the observation of one photon event above $10^{11.3} \mathrm{GeV}$ in five years of data collection) of Probe of Extreme Multi-Messenger Astrophysics (POEMMA). The previous limit on $\tau_{X}$ derived in Ref. [77] is also shown for comparison.

a detailed description of the photon directional exposure to the Auger Collaboration. On the other hand, it is important to note that the contribution from the nucleon flux to the allparticle intensity would tend to compensate any possible reduction in the photon exposure. Indeed, we can derive a lower limit on $\tau_{X}$ using only the nucleon flux expected from the $X$-decay. A rough estimate of such a limit can be obtained through a rescaling of the results shown in Fig. 3 by the $\gamma / N$ ratio. An additional compensation can be picked up by using also the Telescope Array (TA) observations. TA has accumulated an exposure of approximately $8,300 \mathrm{~km}^{2} \mathrm{sryr}$ without observation of events above $10^{11.3} \mathrm{GeV}$ [89]. After removing the band of declination common to both experiments, this becomes an approximately $10 \%$ effect.

\section{POEMMA DISCOVERY REACH}

In line with our stated plan, we now estimate the sensitivity of next generation UHECR experiments to detect signals of WIMPzillas. At present, the most advanced concept in pursuit of this objective is POEMMA [90]. POEMMA will comprise two satellites flying in loose formation at $525 \mathrm{~km}$ altitudes, with stereoscopic UHECR observation mode and monocular Earthlimb viewing mode. In stereo fluorescence mode, the two detectors view a common immense atmospheric volume corresponding to approximately $10^{13}$ tons of atmosphere. The stereo mode yields roughly an order of magnitude increase in yearly UHECR exposure compared to that obtainable by ground observatory arrays and 2 orders of magnitude compared to ground fluorescence observations. In the limb-viewing mode, POEMMA reaches nearly $10^{10}$ gton. The stereoscopic sensitivity of POEMMA to probe the lifetime of SHDM is shown in Fig. 3. Detection of an extreme-energy photon would be a momentous discovery. If this were the case, POEMMA could be switched into limb mode to rapidly increase statistics.

It is also noteworthy that cosmic-ray showers initiated by extreme-energy photons develop, on average, deeper in the atmosphere than air showers of the same primary energy initiated by protons [91]. This is portrayed through the observable $X_{\max }$, which describes the atmospheric column depth at which the longitudinal development of a cosmicray shower reaches the maximum. Of particular interest here, for energies $E \gtrsim E_{0}$, the average $X_{\max }$ of photon and proton showers differs by more than $100 \mathrm{~g} / \mathrm{cm}^{2}$ [92]. Ergo, while the expected monocular performance of POEMMA to identify the UHECR primary $\left(\Delta X_{\max } \sim 100 \mathrm{~g} / \mathrm{cm}^{2}\right)$ is not as accurate as that for the stereo mode $\left(\Delta X_{\max } \lesssim 30 \mathrm{~g} / \mathrm{cm}^{2}\right)$, it is still sufficient to characterize the $\gamma / N$ ratio.

We now comment on the impact of the adopted DM profile in our calculations. Because we are averaging over the entire field of view of the experiments, the selection of the DM profile carries only a very small effect. This is visible in Fig. 1, where we show that the differences between the DM halo profiles are evident for angles $\theta \lesssim 10^{\circ}$. Indeed, the deviation from our results when considering the Burkert profile rather than the canonical NFW is about $10 \%$. Because the Galactic Center is well within the field of view of Auger, the limit on $\tau_{X}$ is slightly relaxed when considering the Burkert profile. One the other hand, the POEMMA sensitivity that averages over the orbital period is increased.

\section{CONCLUSIONS}

Thus far, the various ongoing efforts to produce or detect WIMPs have not given us any promising clues, and moreover, as of today, there have been no definitive hints for beyond SM physics at any accessible energy scale. This rather unexpected situation has motivated a new approach to understand the particle nature of DM. If the Universe is fine-tuned, then the natural mass range for the dark sector would be the Planck scale. Such SHDM can arise from string theory or other high-energy phenomena, and the observed DM abundance can be successfully produced during the inflationary epoch. We have studied the constraints on SHDM models given by recent UHECR observations. For masses in the range $10^{14} \lesssim M_{X} / \mathrm{GeV} \lesssim 10^{16}$, we derived the strongest (95\% C.L.) limit on the lifetime of hadronically decaying SHDM particles. We also explored the prospects for WIMPzilla discovery with future observations of UHECRs. We end with an observation: in five years of data collection, POEMMA (in the limb-viewing mode) will have the potential to accumulate an unprecedented exposure (approximately $10^{6} \mathrm{~km}^{2} \mathrm{sryr}$ ) and become the ultimate WIMPzilla hunter. 


\section{ACKNOWLEDGMENTS}

We are thankful to Mikhail Kuznetsov for insightful remarks on the manuscript and providing the normalization for the photon flux expected to be observed at the declination of the Pierre Auger Observatory. We also thank Sergey Troitsky as well as our colleagues of the Pierre Auger and POEMMA collaborations for some valuable discussions. This work has been supported by the by the U.S. National Science Foundation (Grant No. PHY1620661) and the NASA (Grant No. 80NSSC18K0464). Any opinions, findings, and conclusions or recommendations expressed in this material are those of the authors and do not necessarily reflect the views of the NSF or NASA.
[1] G. Bertone, D. Hooper, and J. Silk, Particle dark matter: Evidence, candidates and constraints, Phys. Rep. 405, 279 (2005).

[2] J. L. Feng, Dark matter candidates from particle physics and methods of detection, Annu. Rev. Astron. Astrophys. 48, 495 (2010).

[3] B. W. Lee and S. Weinberg, Cosmological Lower Bound on Heavy Neutrino Masses, Phys. Rev. Lett. 39, 165 (1977).

[4] M. I. Vysotsky, A. D. Dolgov, and Y. B. Zeldovich, Cosmological restriction on neutral lepton masses, Pis'ma Zh. Eksp. Teor. Fiz. 26, 200 (1977) [JETP Lett. 26, 188 (1977)].

[5] H. Goldberg, Constraint on the Photino Mass from Cosmology, Phys. Rev. Lett. 50, 1419 (1983); Erratum, Phys. Rev. Lett. 103, 099905(E) (2009).

[6] G. Steigman and M. S. Turner, Cosmological constraints on the properties of weakly interacting massive particles, Nucl. Phys. B253, 375 (1985).

[7] P. Gondolo and G. Gelmini, Cosmic abundances of stable particles: Improved analysis, Nucl. Phys. B360, 145 (1991).

[8] G. Steigman, B. Dasgupta, and J. F. Beacom, Precise relic WIMP abundance and its impact on searches for dark matter annihilation, Phys. Rev. D 86, 023506 (2012).

[9] K. Griest and M. Kamionkowski, Unitarity Limits on the Mass and Radius of Dark Matter Particles, Phys. Rev. Lett. 64, 615 (1990).

[10] K. Blum, Y. Cui, and M. Kamionkowski, An ultimate target for dark matter searches, Phys. Rev. D 92, 023528 (2015).

[11] O. Buchmueller, C. Doglioni, and L. T. Wang, Search for dark matter at colliders, Nat. Phys. 13, 217 (2017).

[12] B. Penning, The pursuit of dark matter at colliders: An overview, J. Phys. G 45, 063001 (2018).

[13] T. Marrodn Undagoitia and L. Rauch, Dark matter directdetection experiments, J. Phys. G 43, 013001 (2016).

[14] E. Aprile et al. (XENON Collaboration), First Dark Matter Search Results from the XENON1T Experiment, Phys. Rev. Lett. 119, 181301 (2017).

[15] D. S. Akerib et al. (LUX Collaboration), Results from a Search for Dark Matter in the Complete LUX Exposure, Phys. Rev. Lett. 118, 021303 (2017).

[16] X. Cui et al. (PandaX-II Collaboration), Dark Matter Results from 54-Ton-Day Exposure of PandaX-II Experiment, Phys. Rev. Lett. 119, 181302 (2017).

[17] C. Amole et al. (PICO Collaboration), Dark Matter Search Results from the PICO-60 $\mathrm{C}_{3} \mathrm{~F}_{8}$ Bubble Chamber, Phys. Rev. Lett. 118, 251301 (2017).
[18] M. G. Aartsen et al. (IceCube Collaboration), Search for neutrinos from dark matter self-annihilations in the center of the Milky Way with 3 years of IceCube/DeepCore, Eur. Phys. J. C 77, 627 (2017).

[19] A. Albert et al., Results from the search for dark matter in the Milky Way with 9 years of data of the ANTARES neutrino telescope, Phys. Lett. B 769, 249 (2017).

[20] M. L. Ahnen et al. (MAGIC and Fermi-LAT Collaborations), Limits to dark matter annihilation cross-section from a combined analysis of MAGIC and Fermi-LAT observations of dwarf satellite galaxies, J. Cosmol. Astropart. Phys. 02 (2016) 039.

[21] H. Abdallah et al. (HESS Collaboration), Search for $\gamma$-Ray Line Signals from Dark Matter Annihilations in the Inner Galactic Halo from 10 Years of Observations with H.E.S.S., Phys. Rev. Lett. 120, 201101 (2018).

[22] A. Albert et al. (HAWC Collaboration), Dark matter limits from dwarf spheroidal galaxies with the HAWC gamma-ray observatory, Astrophys. J. 853, 154 (2018).

[23] A. U. Abeysekara et al. (HAWC Collaboration), A search for dark matter in the Galactic halo with HAWC, J. Cosmol. Astropart. Phys. 02 (2018) 049.

[24] M. Ackermann et al. (Fermi-LAT Collaboration), Constraints on the Galactic halo dark matter from Fermi-LAT diffuse measurements, Astrophys. J. 761, 91 (2012).

[25] D. J. H. Chung, E. W. Kolb, and A. Riotto, Superheavy dark matter, Phys. Rev. D 59, 023501 (1998).

[26] V. Kuzmin and I. Tkachev, Ultrahigh-energy cosmic rays, superheavy long living particles, and matter creation after inflation, Pis'ma Zh. Eksp. Teor. Fiz. 68, 255 (1998) [JETP Lett. 68, 271 (1998)].

[27] V. Kuzmin and I. Tkachev, Matter creation via vacuum fluctuations in the early universe and observed ultrahighenergy cosmic ray events, Phys. Rev. D 59, 123006 (1999).

[28] E. W. Kolb, D. J. H. Chung, and A. Riotto, WIMPzillas, AIP Conf. Proc. 484, 91 (1999).

[29] D. J. H. Chung, E. W. Kolb, A. Riotto, and I. I. Tkachev, Probing Planckian physics: Resonant production of particles during inflation and features in the primordial power spectrum, Phys. Rev. D 62, 043508 (2000).

[30] V. A. Kuzmin and I. I. Tkachev, Ultrahigh-energy cosmic rays and inflation relics, Phys. Rep. 320, 199 (1999).

[31] D. J. H. Chung, P. Crotty, E. W. Kolb, and A. Riotto, On the gravitational production of superheavy dark matter, Phys. Rev. D 64, 043503 (2001). 
[32] E. W. Kolb, A. A. Starobinsky, and I. I. Tkachev, TransPlanckian WIMPzillas, J. Cosmol. Astropart. Phys. 07 (2007) 005.

[33] K. Kannike, A. Racioppi, and M. Raidal, Super-heavy dark matter: Towards predictive scenarios from inflation, Nucl. Phys. B918, 162 (2017).

[34] S. Rappoccio, The experimental status of direct searches for exotic physics beyond the standard model at the Large Hadron Collider, Rev. Phys. 4, 100027 (2019).

[35] S. Weinberg, Anthropic Bound on the Cosmological Constant, Phys. Rev. Lett. 59, 2607 (1987).

[36] V. Agrawal, S. M. Barr, J. F. Donoghue, and D. Seckel, Anthropic Considerations in Multiple Domain Theories and the Scale of Electroweak Symmetry Breaking, Phys. Rev. Lett. 80, 1822 (1998).

[37] L. Susskind, The anthropic landscape of string theory, Universe or Multiverse, edited by B. Carr, p. 247.

[38] B. Feldstein, L. J. Hall, and T. Watari, Landscape prediction for the higgs boson and top quark masses, Phys. Rev. D 74, 095011 (2006).

[39] J. F. Donoghue, K. Dutta, A. Ross, and M. Tegmark, Likely values of the Higgs vev, Phys. Rev. D 81, 073003 (2010).

[40] M. R. Douglas, The statistics of string / M theory vacua, J. High Energy Phys. 05 (2003) 046.

[41] S. Ashok and M. R. Douglas, Counting flux vacua, J. High Energy Phys. 01 (2004) 060.

[42] F. Wilczek, A model of anthropic reasoning, addressing the dark to ordinary matter coincidence, Universe or Multiverse, edited by B. Card, p. 151.

[43] S. Hellerman and J. Walcher, Dark matter and the anthropic principle, Phys. Rev. D 72, 123520 (2005).

[44] M. Tegmark, A. Aguirre, M. Rees, and F. Wilczek, Dimensionless constants, cosmology and other dark matters, Phys. Rev. D 73, 023505 (2006).

[45] B. Freivogel, Anthropic explanation of the dark matter abundance, J. Cosmol. Astropart. Phys. 03 (2010) 021.

[46] D. J. H. Chung, E. W. Kolb, A. Riotto, and L. Senatore, Isocurvature constraints on gravitationally produced superheavy dark matter, Phys. Rev. D 72, 023511 (2005).

[47] P. A. R. Ade et al. (Planck Collaboration), Planck 2015 results XX: Constraints on inflation, Astron. Astrophys. 594, A20 (2016).

[48] P. A. R. Ade et al. (BICEP2 and Keck Array Collaborations), Improved Constraints on Cosmology and Foregrounds from BICEP2 and Keck Array Cosmic Microwave Background Data with Inclusion of $95 \mathrm{GHz}$ Band, Phys. Rev. Lett. 116, 031302 (2016).

[49] M. Garny, M. Sandora, and M. S. Sloth, Planckian Interacting Massive Particles as Dark Matter, Phys. Rev. Lett. 116, 101302 (2016).

[50] V. Berezinsky, M. Kachelriess, and A. Vilenkin, UltrahighEnergy Cosmic Rays without GZK Cutoff, Phys. Rev. Lett. 79, 4302 (1997).

[51] V. A. Kuzmin and V. A. Rubakov, Ultrahigh-energy cosmic rays: A Window to postinflationary reheating epoch of the universe, Phys. At. Nucl. 61, 1028 (1998).

[52] A. Aab et al. (Pierre Auger Collaboration), The Pierre Auger Observatory: Contributions to the 35th International Cosmic Ray Conference (ICRC 2017), arXiv:1708.06592.
[53] G. J. Feldman and R. D. Cousins, A unified approach to the classical statistical analysis of small signals, Phys. Rev. D 57, 3873 (1998).

[54] A. Aab et al. (Pierre Auger Collaboration), Search for photons with energies above $10^{18} \mathrm{eV}$ using the hybrid detector of the Pierre Auger Observatory, J. Cosmol. Astropart. Phys. 04 (2017) 009.

[55] R. U. Abbasi et al. (Telescope Array Collaboration), Constraints on the diffuse photon flux with energies above $10^{18} \mathrm{eV}$ using the surface detector of the Telescope Array experiment, Astropart. Phys. 110, 8 (2019).

[56] J. F. Navarro, C. S. Frenk, and S. D. M. White, A Universal density profile from hierarchical clustering, Astrophys. J. 490, 493 (1997).

[57] J. F. Navarro, A. Ludlow, V. Springel, J. Wang, M. Vogelsberger, S. D. M. White, A. Jenkins, C. S. Frenk, and A. Helmi, The diversity and similarity of cold dark matter halos, Mon. Not. R. Astron. Soc. 402, 21 (2010).

[58] J. Einasto, Kinematics and dynamics of stellar systems, Tr. Astrofiz. Inst. Alma-Ata 5, 87 (1965).

[59] A. Burkert, The structure of dark matter halos in Dwarf Galaxies, IAU Symp. 171, 175 (1996); Astrophys. J. 447, L25 (1995).

[60] B. Moore, S. Ghigna, F. Governato, G. Lake, T. R. Quinn, J. Stadel, and P. Tozzi, Dark matter substructure within galactic halos, Astrophys. J. 524, L19 (1999).

[61] M. Tanabashi et al. (Particle Data Group), Review of particle physics, Phys. Rev. D 98, 030001 (2018).

[62] M. Cirelli, G. Corcella, A. Hektor, G. Hütsi, M. Kadastik, P. Panci, M. Raidal, F. Sala, and A. Strumia, PPPC 4 DM ID: A poor particle physicist cookbook for dark matter indirect detection, J. Cosmol. Astropart. Phys. 03 (2011) 051; Erratum, J. Cosmol. Astropart. Phys. 10 (2012) E01(E).

[63] V. Berezinsky and M. Kachelriess, Limiting SUSY QCD spectrum and its application for decays of superheavy particles, Phys. Lett. B 434, 61 (1998).

[64] M. Birkel and S. Sarkar, Extremely high-energy cosmic rays from relic particle decays, Astropart. Phys. 9, 297 (1998).

[65] V. Berezinsky and M. Kachelriess, Monte Carlo simulation for jet fragmentation in SUSY QCD, Phys. Rev. D 63, 034007 (2001).

[66] S. Sarkar and R. Toldra, The High-energy cosmic ray spectrum from relic particle decay, Nucl. Phys. B621, 495 (2002).

[67] C. Barbot and M. Drees, Detailed analysis of the decay spectrum of a super heavy $X$ particle, Astropart. Phys. 20, 5 (2003).

[68] V. N. Gribov and L. N. Lipatov, $e^{+} e^{-}$pair annihilation and deep inelastic ep scattering in perturbation theory, Yad. Fiz. 15, 1218 (1972) [Sov. J. Nucl. Phys. 15, 675 (1972)].

[69] V. N. Gribov and L. N. Lipatov, Deep inelastic ep scattering in perturbation theory, Yad. Fiz. 15, 781 (1972) [Sov. J. Nucl. Phys. 15, 438 (1972)].

[70] Y. L. Dokshitzer, Calculation of the structure functions for deep inelastic scattering and $e^{+} e^{-}$annihilation by perturbation theory in quantum chromodynamics, Zh. Eksp. Teor. Fiz. 73, 1216 (1977) [Sov. Phys. JETP 46, 641 (1977)].

[71] G. Altarelli and G. Parisi, Asymptotic freedom in parton language, Nucl. Phys. B126, 298 (1977). 
[72] S. L. Dubovsky and P. G. Tinyakov, Galactic anisotropy as signature of CDM related ultrahigh-energy cosmic rays, JETP Lett. 68, 107 (1998).

[73] N. W. Evans, F. Ferrer, and S. Sarkar, The Anisotropy of the ultrahigh-energy cosmic rays, Astropart. Phys. 17, 319 (2002).

[74] R. Aloisio and F. Tortorici, Super heavy dark matter and UHECR anisotropy at low energy, Astropart. Phys. 29, 307 (2008).

[75] O. E. Kalashev and M. Y. Kuznetsov, Heavy decaying dark matter and large-scale anisotropy of high-energy cosmic rays, Pis'ma Zh. Eksp. Teor. Fiz. 106, 65 (2017) [JETP Lett. 106, 73 (2017)].

[76] R. Aloisio, V. Berezinsky, and M. Kachelriess, On the status of superheavy dark matter, Phys. Rev. D 74, 023516 (2006).

[77] O. K. Kalashev and M. Y. Kuznetsov, Constraining heavy decaying dark matter with the high energy gamma-ray limits, Phys. Rev. D 94, 063535 (2016).

[78] A. Aab et al. (Telescope Array and Pierre Auger Collaborations), Searches for large-scale anisotropy in the arrival directions of cosmic rays detected above energy of $10^{19} \mathrm{eV}$ at the Pierre Auger Observatory and the Telescope Array, Astrophys. J. 794, 172 (2014).

[79] M. Kuznetsov (private communication).

[80] R. Aloisio, S. Matarrese, and A. V. Olinto, Super heavy dark matter in light of BICEP2, Planck and ultra high energy cosmic rays observations, J. Cosmol. Astropart. Phys. 08 (2015) 024.

[81] V. Berezinsky and A. A. Mikhailov, Anisotropy of ultrahigh-energy cosmic rays in the halo models, Phys. Lett. B 449, 237 (1999).

[82] P. Gondolo, G. Gelmini, and S. Sarkar, Cosmic neutrinos from unstable relic particles, Nucl. Phys. B392, 111 (1993).
[83] A. Esmaili, A. Ibarra, and O. L. G. Peres, Probing the stability of superheavy dark matter particles with highenergy neutrinos, J. Cosmol. Astropart. Phys. 11 (2012) 034.

[84] M. Y. Kuznetsov, Hadronically decaying heavy dark matter and high-energy neutrino limits, JETP Lett. 105, 561 (2017).

[85] M. Kachelriess, O.E. Kalashev, and M. Y. Kuznetsov, Heavy decaying dark matter and IceCube high energy neutrinos, Phys. Rev. D 98, 083016 (2018).

[86] M. Garny, A. Palessandro, M. Sandora, and M. S. Sloth, Theory and phenomenology of Planckian interacting massive particles as dark matter, J. Cosmol. Astropart. Phys. 02 (2018) 027.

[87] O. E. Kalashev, G. I. Rubtsov, and S. V. Troitsky, Sensitivity of cosmic-ray experiments to ultra-high-energy photons: Reconstruction of the spectrum and limits on the superheavy dark matter, Phys. Rev. D 80, 103006 (2009).

[88] A. Aab et al. (Pierre Auger Collaboration), The Pierre Auger Observatory: Contributions to the 34th International Cosmic Ray Conference (ICRC 2015), arXiv:1509.03732 [Proc. Sci. (ICRC2015) (to be published)].

[89] Telescope Array and Pierre Auger Collaborations, Pierre Auger Observatory and Telescope Array: Joint contributions to the 35th International Cosmic Ray Conference (ICRC 2017), arXiv:1801.01018.

[90] A. V. Olinto et al., POEMMA: Probe of extreme multimessenger astrophysics, Proc. Sci. ICRC2017 (2018) 542.

[91] L. A. Anchordoqui, Ultra-high-energy cosmic rays, Phys. Rep. 801, 1 (2019).

[92] M. Risse and P. Homola, Search for ultrahigh energy photons using air showers, Mod. Phys. Lett. A 22, 749 (2007). 